\title{
Efficacy of Hyaluronidase in Enhancing the Effect of Inferior Alveolar Nerve Block when Used in Conjugation with Lidocain
}

\author{
Dr. Neelkamal ${ }^{1}$, Dr. Ajay Chhabra ${ }^{2}$, Dr. Varun Jindal, Dr. Simranpal Singh Bindra \\ Bhojia Dental College and Hospital, Bhud., distt. Nalagard, Himachal Pradesh
}

\begin{abstract}
Aim: Present study was conducted to assess the efficacy of hyaluronidase in enhancing the effect of inferior alveolar nerve block when used in conjugation with lidocain. Methods: Patients diagnosed with irreversible pulpitis in mandibular molars were selected for study. After noting the baseline stimulus on premolars by electronic pulp tester, inferior alveolar nerve block was injected. Thirty minutes after the onset of anesthesia placebo and hyaluronidase was injected during the control and test phase of the study respectively. Duration of the anesthesia was recorded with the help of electronic pulp tester on the premolar and by needle prick on the gingiva. Results: Significant increase in the pulpal and gingival anesthesia was observed by administering hyaluronidase after the lidocain injection. Conclusion: Hyaluronidase when used before the end of anesthetic effect of lidocain in inferior alveolar nerve blocks can increase the efficacy of anesthetic.
\end{abstract}

Keywords: hyalyronidase, lidocain, inferior, alveolar, nerve, block

\section{Introduction}

The discovery in the late 1800 s of a group of chemicals with ability to prevent pain without inducing loss of consciousness was one of the major steps in the advancements of the medical and dental professions. For the first time, medical and dental procedures, could be carried out easily and in the absence of pain, a fat that is virtually taken for granted by contemporary medical and dental professionals and their patients. ${ }^{2}$

Local anesthesia has been defined as loss of sensation in a circumscribed area o the body caused by depression of excitation of the conduction process in peripheral nerves. ${ }^{1}$ The concept behind the local anaestheisa is simple: They prevent both the generation and conduction of nerve impulse. Therefore the absorbed impulse, prevented from reaching the brain, cannot be interpreted by the patient as pain. $^{2}$

The options among long-term local anesthesias used in dentistry are very restricted, and currently there are no longterm local anesthesias available that can be safely used with medically compromised patients. Therefore, to increase the duration of action, Local anaesthesias with intermediate duration such as lidocaine, articaine, and prilocaine can be combined with the hyaluronidase enzyme. ${ }^{3}$

Therapeutically, this enzyme is used as a comedication with LAs and drugs with pain-reducing behavior. The term hyaluronidase was introduced by Meyer et al. ${ }^{4}$ The first therapeutic use of this enzyme was mentioned in 1928 as a so-called spreading factor, which facilitates the uptake of subcutaneously applied vaccines and toxins. Hyaluronidase is an enzyme derived from bovine testes, and it exhibits hydrolytic and transglycosidic effects via the splitting of the b-1, 4-glycosidic bond. Hyaluronidase enzymes are able to degrade hyaluronic acid, chondroitin-4- and -6 sulfate, and dermatan sulfate, which are the main components of the extracellular matrix. ${ }^{5}$
However hyaluronidase was not found to improve the success of lidocain anesthesia in a recent double-blind randomized clinical study. ${ }^{6}$ The present study aimed to assess the effect of hyaluronidase on the duration of lidocain anesthesia when used for inferior alveolar nerve block before the reversal of anesthesia.

\section{Materials and Method}

The present study was approved by the ethical comedy of the Bhojia Dental College and Hospital, Baddi, Himachal Pradesh, India. Twenty patients aged from 19 to 53 years suffering from chronic irreversible pulpitis of mandibular molar having intact premolars adjacent to the affected tooth were included for the study. After explaining the procedure, informed consent was taken from the patients. Patch test was performed over the patients, both for the local anesthetic solution and hyaluronidase to rule out any kind of allergy for the drugs.

After the recording the baseline stimulus over the premolar tooth local anesthetic was administered $(2 \mathrm{ml}$ of lidocain solution with 1:100000 adrenalin) to block the inferior alveolar nerve. Subsequent to the anesthetic injection, stimuli (by electronic pulp tester) were applied every 2 minutes until they reached a value of 80 which was considered to be the onset of profound anesthesia. Thirty minutes after the profound anesthesia a placebo $(1 \mathrm{ml}$ of saline) was injected and the duration of anesthesia was noted by checking it with electric stimuli over the adjacent premolar tooth after every 10 minutes. Anesthesia was considered to be profound until the score remained 80 .

The duration of the anesthetic effect on the soft tissue was evaluated using a nociceptive mechanical stimulus (pinprick) using a 25-G needle in the buccal gingiva around the first and second lower premolars. These stimuli were performed in the same intervals as the electrical stimuli. The duration of the anesthetic effect in the gingiva was considered to be the interval from the loss of sensation to the 


\section{International Journal of Science and Research (IJSR) \\ ISSN (Online): 2319-7064}

Index Copernicus Value (2013): 6.14 | Impact Factor (2014): 5.611

mechanical stimulus until the return of the pinprick sensation.

In the next appointment same procedure was performed except that this time 30 minutes after the profound anesthesia, $1 \mathrm{ml}$ of $75 \mathrm{IU}$ of hyaluronidase was administered and the duration of anesthesia was recorded.

\section{Statistical Analysis}

Data analysis was performed using SPSS 16.0 software. Comparisons between the control and experimental phase were performed using the Student $\mathrm{t}$ test. $\mathrm{P}$ value $<.05$ was considered to be significant. (Table 1)

\section{Results}

Lidocaine with hyaluronidase significantly increased pulpal and gingival anesthesia.

\section{Discussion}

Following administration of a local anaesthetic into soft tissues near a nerve, molecules of the local anesthetic transverse the distance from one site to another according to their concentration gradient. During the induction phase of the anesthesia, the local anesthetic moves from extraneural site of deposition toward the nerve (as well as in all other possible directions). This process is called as diffusion. Penetration of an anatomic barrier occurs when a drug passes through a tissue that tends to restrict free molecular movement. ${ }^{2}$

The role of hyaluronidase here is that it increases the diffusablitiy of the local anesthetic through tissues so as to reach it to nerve, therefore when it is used in conjugation with LA solution, it might fasten onset of anesthetic effect as observed in the previous studies. ${ }^{7,8}$ However, the present study aimed to increase the duration of the anesthesia as the hyaluronidase was not administered in conjugation with LA, rather it was injected thirty minutes after the onset of anesthesia.

When the local anesthetic is administered it diffuses in all the directions. A portion of the injected local anesthetic diffuses towards the nerve and into the nerve. However, a significant portion of the injected drug also diffuses away from the nerve into the tissue. This solution is present in the tissues until it is completely absorbed up in the blood. ${ }^{2}$ Injection of hyaluronidase 30 minutes after the onset of anesthesia might have increased the diffusion of the LA present in the tissues and directed it towards the nerve.

Our findings show that hyaluronidase injected before the end of anesthesia significantly prolongs the duration of nerve blockade compared with the control group (LA without hyaluronidase) in both tissues. The duration of pulpal anesthesia was increased by 27.5 minutes in the experimental group. In the soft tissue, the hyaluronidase group also showed an increased duration of anesthesia compared with the placebo group (ie, an increase of 26.2 minutes)
The question whether the findings of the present study were because of the mere introduction of the liquid after the onset of the anesthesia which might have caused the movement of LA toward the nerve is also excluded as the control phase of the study did not show any increase in the duration of anesthesia.

The addition of hyaluronidase, which has a $\mathrm{pH}$ of 6.5 and an optimal $\mathrm{pH}(\mathrm{pKa})$ between 6.4 and 7.4, may decrease the $\mathrm{pH}$ of the tissue surrounding the nerve because the $\mathrm{pH}$ of normal tissue is approximately 7.4. Thus, increasing the amount of the ionized form (cation) and reducing the amount of the nonionized base form ${ }^{3}$ of the LA would make it difficult for the compound to cross the membrane. If the LA and hyaluronidase injections were concomitant, the onset of the block may occur later when the acidic $\mathrm{pH}$ of the tissue requires buffering. The onset of the effect will be directly related to the amount of LA that exists in the base form. However, in our study, at the time point at which the hyaluronidase was injected (ie, 30 minutes after inducing ongoing pulpal anesthesia), even the amount of nonionized LA would have been reduced by the acidic $\mathrm{pH}$. Nevertheless, there was most likely a sufficient amount of LA available because the intensity or "depth" of anesthesia was not affected. In addition, the LA used was lidocaine, which has a low pKa (7.7) compared with those of other LAs. This $\mathrm{pKa}$ is close to the tissue $\mathrm{pH}$ of $7.4,{ }^{2}$ possibly allowing a sufficient proportion of nonionized LA to diffuse into the nerve even though the tissue $\mathrm{pH}$ level may have decreased after the injection of hyaluronidase.

In a previous similar study ${ }^{9}$ the subject chosen for control group and test group were different. In our study subjects were first subjected to control phase and then they were subjected to test phase of study. This prevented the intersubject bias as different patients may have different pain perception physiology.

\section{Conclusion}

Under the limitation of present study it is concluded that the hyaluronidase administered before the end of anesthesia may enhance the duration of anesthesia.

\section{References}

[1] Covino BG, Vasallo HG: Local anesthetics: Mechanisms of action and clinical use, New York, 1976, Grune and Stratton.

[2] Malamed SF. Handbook of Local Anesthesia, 5th ed. St Louis, MO: Mosby; 2004

[3] Tempestini Horliana AC, de Brito MA, Perez FE et al. Hyaluronidase increases the duration of mepivacaine in inferior alveolar nerve blocks. J Oral Maxillofac Surg. 2008;66:286-90.

[4] Schulze C, Bittorf T, Walzel $\mathbf{H}$ et al. Experimental evaluation of hyaluronidase activity in combination with specific drugs applied in clinical techniques of interventional pain management and local anaesthesia. Pain Physician 2008;11:877-83.

[5] Sarvela J, Nikki P. Hyaluronidase improves regional ophthalmic anaesthesia with etidocaine. Can J Anaesth 2000;39:920-4. 


\section{International Journal of Science and Research (IJSR) \\ ISSN (Online): 2319-7064}

Index Copernicus Value (2013): 6.14 | Impact Factor (2014): 5.611

[6] Ridenour S, Reader A. Beck M, Weaver J. Anesthetic efficacy of a combination of hyaluronidase and lidocain with epinephrine in inferior alveolar nerve blocks. Anesthetic Prog 2001;48(1):9-15.

[7] Looby J, Kirby C. Use of hyaluronidase with local anesthetic agents in dentistry. J Am Dent Assoc 1949;38:1-4.

[8] Kirby C, Eckenhoff, Lobby J. The use of hyaluronidase with local anesthetic agents in nerve blocks and infiltration anesthesia. Surgery 1949;25:101-3
[9] Sarvepalli Venkata Satish,Krishna Prasad Shetty, Krishnarao Kilaru, Puridi Bhargavi, E. Srinivas Reddy and Aditya Bellutgi. Comparative Evaluation of the Efficacy of 2\% Lidocaine Containing $\backslash 1: 200,000$ Epinephrine with and without Hyaluronidase (75 IU) in Patients with Irreversible Pulpitis. J Endod 2013;39:1116-1118.

Table 1: The Duration of Pulpal and Gingival Anesthesia in the Control and Experimental Groups

\begin{tabular}{|l|c|c|c|c|}
\hline \multicolumn{1}{|c|}{ Time } & Groups & Control & Experimental Difference & P value \\
Duration of anesthesia (min) & $50.45+/-2.69$ & $85.70+/-5.78$ & 37.25 & $<.0001$ \\
Duration of treatment & $70.04+/-6.32$ & $77.65+/-4.65$ & 2.01 & .22 \\
Duration of gingival anesthesia & $68.15+/-5.04$ & $91.40+/-6.25$ & 24.25 & $<.0001$ \\
\hline
\end{tabular}

